and fibrosis in myocardium, thus delay the progress of the diabetic cardiomyopathy.

\section{Q0041 VASOMOTOR FUNCTION FOLLOWING NEWER GENERATION OF BARE METAL STENT OVERSTRETCH IN A PORCINE CORONARY MODEL}

doi:10.1136/hrt.2010.208967.41

${ }^{1}$ Jing Chen, ${ }^{2}$ Takamitsu Nakamura, Daisuke Matsumoto, Nicolas Chronos, Dongming Hou. ${ }^{1}$ Renmin Hospital, of Wuhan University, Wuhan, China; ${ }^{2}$ Saint Joseph's Translational Research Institute/Saint Joseph's Hospital, Atlanta, Georgia, USA

Backgrounds Overstretch damage after bare metal stent (BMS) placement could trigger cell proliferation and in-stent restenosis (ISR). Newer Co-Cr BMS has thinner stent struts, which designs to minimise cellular response to injury. We aimed to investigate neointimal growth, as well as vasomotor function after overstretch using Co-Cr BMS in a pig coronary model.

Methods 15 vessels in five pigs were assigned to receive BMS (stent struts $91 \mu \mathrm{m}$ ) implantation with either S/A ratio 1.3 (group $\mathrm{I}, \mathrm{n}=7$ ) or 1.5 (group II, $\mathrm{n}=8$ ). Quantitative coronary angiography (OCA) and optical coherence tomography (OCT) were performed at 14 days after stent implantation. Coronary vasomotor function was evaluated by incremental acetylcholine (Ach) $\left(10^{-7}\right.$ and $\left.10^{-6} \mathrm{M}\right)$ and

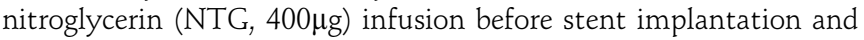
at 14 days. Endothelial response to Ach was measured at 5-10 mm distal to the stent edge.

Results Both OCA and OCT showed that in-stent stenosis of group I were significantly smaller than group II at 14 days (QCA-late loss (LL), $1.22 \pm 0.21 \mathrm{~mm}$ vs $1.79 \pm 0.17 \mathrm{~mm}$; OCT \% AS, $17.0 \pm 7.9 \%$ vs $26.9 \pm 10.7 \%$ at 14 days, $p<0.05$ and 0.001 , respectively). Liner regression analysis QCA-LL is proportional to obtained S/A ratio $(\mathrm{r}=0.60, \mathrm{p}<0.05)$. Endothelium-dependent vasomotion at distal non-stented reference segments was no difference between groups. The mean coronary diameter changes at Ach $10^{-7} \mathrm{M}$ and $10^{-6} \mathrm{M}$ was $2.1 \% \pm 0.2 \%$ and $2.1 \% \pm 0.2 \%$ in goup I; $2.2 \% \pm 0.2 \%$ and $2.1 \% \pm$ $0.2 \%$ in group II ( $p>0.05$, accordingly). There was also no difference before and at 14 days after stent implantation.

Conclusion The progression of neointimal hyperplasia after BMS implantation is positively associated with the extent of coronary artery injury. Coronary endothelial function is preserved after BMS implantation at 14 days, which is independently of overstretch degree.

\section{e0042 THE EFFECT OF ISCHAEMIC POSTCONDITIONING ON THE STRUCTURE, FUNCTION AND CX43 OF MITOCHONDRIA IN RABBIT MYOCARDIAL ISEHEMIA/REPERFUSION INJURY}

doi:10.1136/hrt.2010.208967.42

Yan He, Zhi-Yu Zeng, Guo-Qiang Zhong, Wei Li, Wei-Ke Li. Department of Cardiology, The First Affiliated Hospital of Guangxi Medical University

Objective To investigate the effects of ischaemic postconditioning on structural and functional and connexin 43(Cx43) changes of mitochondria induced by myocardial ischaemia reperfusion (IR) injury of rabbits in vivo and potential mechanism.

Methods In anaesthetised open-chest rabbits, the left anterior descending artery (LAD) was occluded for $30 \mathrm{~min}$ and reperfused for $4 \mathrm{~h}$. Sixty-four rabbits were randomly divided into four groups ( $n=16$ in each group): Sham operation group (Group Sham), ischaemic reperfusion group (Group IR), ischaemic preconditioning group (Group IP) and ischaemic postconditioning group (Group PC) with sixteen rabbits in each. All rabbits in the four groups were killed $4 \mathrm{~h}$ after reperfusion. Myocardial infarct size were determined at the end of the experiment. Mitochondria were isolated with different centrifugations. Ultrastructural changes of mitochondria were observed under electronmicroscope and mitochondrial membrane potential, $\mathrm{Ca}^{2+}$ concentration, MDA content and SOD activity of myocardial mitochondria were examined. The content of the mitochondria Cx43 were detected with Western Blot.

Results Myocardial infarct size was significantly reduced in IP $(18.9 \pm 2.8) \%)$ and PC $(19.1 \pm 3.9) \%)$ groups as compared to IR groups $(35.7 \pm 5.8) \%, p<0.01)$. Compared with group IR, the damage of mitoehondrial ultrastrueture were milder and $\mathrm{Ca}^{2+}$ concentration and MDA content were much lower in group IP and group PC $(p<0.05)$. Mitochondrial membrane potential $(p<0.01)$ and SOD activity of myocardial mitochondria in group IP and group PC was significantly higher than that in group IR $(p<0.05)$. Compared with sham group, the mitochondria $\mathrm{Cx} 43$ expression is distinctly decreased compared group IR $(\mathrm{p}<0.05)$ and no significant difference was found between Group IP and Group PC.

Conclusion PC can protect mitochondrial ultrastructure by increaseing mitochondrial membrane potential and SOD activity, and by alleviating $\mathrm{Ca}^{2+}$ overload, and by decreasing MDA content in myocardial mitochondria. The mechanism of $\mathrm{PC}$ protection to mitochondria may be concerned with PC attenuating the decrease the mitochondria $\mathrm{Cx} 43$ expression induced by ischaemia/reperfusion injury.

\section{e0043 EFFECTS OF SIMVASTATIN ON ANGIOGENESIS AND THE EXPRESSION OF ANG1 AFTER MYOCARDIAL INFARCTION IN RATS}

doi:10.1136/hrt.2010.208967.43

He Quan, Qin Shu, Ma Kanghua, Luo Suxin, Zhang Xiaogang. The First Affiliated Hospital Chongqing Medical University

Objective To investigate the effects of simvastatin on myocardial angiogenesis and the expression of angiopoietin- 1 after experimental myocardial infarction (MI) in rats.

Methods 60 healthy adult SD rats were randomly divided into the sham operated group, the control group, low dose of simvastatin $\left(1 \mathrm{mg} \cdot \mathrm{kg}^{-1} \cdot \mathrm{d}^{-1}\right)$ group, medium dose of simvastatin $(10 \mathrm{mg}$. $\left.\mathrm{kg}^{-1} \cdot \mathrm{d}^{-1}\right)$ group, high dose of simvastatin $\left(40 \mathrm{mg} \cdot \mathrm{kg}^{-1} \cdot \mathrm{d}^{-1}\right)$ group. Left anterior descending coronary underwent permanent occlusion to establish the MI model. Rats were administered simvastatin respectively via oral gavage for four consecutive weeks starting at the next day. Density of new microvessels in the ischaemic area, LVMI, protein and mRNA expression of Ang-1 were detected 4 weeks after operation. Results (1) Compared with the control group, the Density of new microvessels in low and medium dose of simvastatin group increased significantly $(p<0.05)$; and those did not changed significantly in high dose of simvastatin group ( $p>0.05)$ (2) LVMI in low and medium dose of simvastatin group decreased significantly compared with that in control group $(p<0.05)$, and further decreased in high dose of simvastatin group. (3) The protein and mRNA expression of Ang-1 in all simvastatin group increased significantly compared with that in control group $(p<0.05)$.

Conclusion (1) Low and medium dose of simvastatin can stimulate myocardial angiogenesis after MI, wherase high dose of simvastatin have no pro-angiogenic effect. (2) the pro-angiogenic effect of simvastatin may be associated with upregulated expression of Ang-1.

\section{e0044 THE ROLE OF ANG1 AND ENOS IN THE PROANGIOGENIC EFFECT OF SIMVASTATIN AFTER MYOCARDIAL INFARCTION IN RATS}

doi:10.1136/hrt.2010.208967.44

He Quan, Qin Shu, Ma Kanghua, Luo Suxin, Zhang Xiaogang. The First Affiliated Hospital Chongqing Medical University

Objective To investigate the roles of angiopoietin-1 (Ang-1) and endothelial nitric oxide synthase (eNOS) in pro-angiogenic 